\title{
Comparing the cancer potencies of emissions from vapourised nicotine products including e-cigarettes with those of tobacco smoke
}

\author{
William E Stephens
}

\begin{abstract}
- Additional material is published online only. To view please visit the journal online (http://dx.doi.org/10.1136/ tobaccocontrol-2017-053808).
\end{abstract}

\section{Correspondence to}

DrWilliam E Stephens, School of Earth \& Environmental Sciences, University of St Andrews, Irvine Building, North Street, St Andrews, Fife KY16 9AL, Scotland, UK;

Ed.Stephens@st-andrews.ac.uk

Received 24 April 2017 Revised 18 July 2017 Accepted 18 July 2017 Published Online First 4 August 2017

\section{Linked}

- http://dx.doi.org/10.1136/ tobaccocontrol-2017-053969

\section{CrossMark}

To cite: Stephens WE. Tob Control 2018;27:10-17.

\begin{abstract}
Background Quantifying relative harm caused by inhaling the aerosol emissions of vapourised nicotine products compared with smoking combustible tobacco is an important issue for public health.
\end{abstract}

Methods The cancer potencies of various nicotinedelivering aerosols are modelled using published chemical analyses of emissions and their associated inhalation unit risks. Potencies are compared using a conversion procedure for expressing smoke and e-cigarette vapours in common units. Lifetime cancer risks are calculated from potencies using daily consumption estimates.

Results The aerosols form a spectrum of cancer potencies spanning five orders of magnitude from uncontaminated air to tobacco smoke. E-cigarette emissions span most of this range with the preponderance of products having potencies $<1 \%$ of tobacco smoke and falling within two orders of magnitude of a medicinal nicotine inhaler; however, a small minority have much higher potencies. These high-risk results tend to be associated with high levels of carbonyls generated when excessive power is delivered to the atomiser coil. Samples of a prototype heat-not-burn device have lower cancer potencies than tobacco smoke by at least one order of magnitude, but higher potencies than most e-cigarettes. Mean lifetime risks decline in the sequence: combustible cigarettes > > heat-not-burn > > e-cigarettes (normal power) $\geq$ nicotine inhaler.

Conclusions Optimal combinations of device settings, liquid formulation and vaping behaviour normally result in e-cigarette emissions with much less carcinogenic potency than tobacco smoke, notwithstanding there are circumstances in which the cancer risks of e-cigarette emissions can escalate, sometimes substantially. These circumstances are usually avoidable when the causes are known.

\section{INTRODUCTION}

Opinion is divided about vapourised nicotine products (VNPs), such as e-cigarettes (ECs) which heat a nicotine-containing liquid and heat-not-burn ( $\mathrm{HnB})$ products which heat tobacco. ${ }^{12}$ The general public tends to view 'vaping' ECs as equally or more harmful than smoking tobacco. ${ }^{34}$ More scientific evidence addressing the factors that lead to harm and, where possible, quantification of their effects is needed to inform scientific debates and address public uncertainty. ${ }^{5}$

Both cigarette smoke and VNP emissions (or 'vapour') are aerosol mixtures of particulates and droplets in gaseous matrices, but each is generated by a different process from different precursors within different temperature ranges. Nicotine is not a carcinogen but VNPs transfer a burden of known carcinogens in the aerosol. Assessing the relative harmfulness of different VNPs requires meaningful indicators of harm. Common approaches include the analysis of biomarkers in bodily fluids, in vivo and in vitro toxicity studies, and evaluating the numbers of individual chemicals that exceed a specified threshold of safety. ${ }^{6-10}$ The health risks of smoking were established half a century ago by epidemiological evidence of associated morbidity and mortality but it may be several years before such approaches can definitively assess the harm of VNPs. ${ }^{11}$ Meanwhile, public health advice depends on quantitative chemical and toxicological approaches to model VNP risks.

This study aims to (1) derive a procedure that overcomes the problem of incompatible emissions data sets, (2) reduce the information on carcinogenic risk represented by multiple individual compounds to a single latent variable (potency) that reflects the cancer risk and (3) calculate cancer potencies from published emissions data and compare various nicotine product risks by factoring in exposure estimates.

\section{METHODOLOGY}

\section{Smoke and vapour emissions}

Compounds from mainstream smoke analyses are typically reported in mass units $(\mathrm{mg}, \mu \mathrm{g}$, $\mathrm{ng}$ as appropriate) per cigarette. Fewer analyses of hazardous compounds in VNPs have been published and the units reported vary widely, usually as mass of compound per volume of vapour (often as $\mu \mathrm{g}$ per $\mathrm{N}$ puffs where $\mathrm{N}$ ranges from 1 to several hundred puffs of fixed volume). A few studies report mass units of toxicant/volume of precursor e-liquid but these are not included in this study.

Different machine smoking protocols purported to simulate human smoking add another complication. The ISO (International Organization for Standardisation) protocol draws $35 \mathrm{~mL}$ per puff of smoke, whereas the Massachusetts Department of Public Health (MDPH) protocol draws $45 \mathrm{~mL}$ and the Health Canada Intense (HCI) (intense) protocol draws $55 \mathrm{~mL}$ (see online supplementary file). ${ }^{12}$ Only volume is considered here, not the withdrawal rate or interval between puffs. The ISO protocol leaves cigarette filter ventilation holes unblocked, whereas MDPH blocks $50 \%$ by taping over half the filter circumference, and HCI blocks these holes entirely. 
Ventilation blocking reduces dilution by air and increases emissions, thus smoking protocol and filter ventilation are fundamental considerations when comparing toxicant concentrations in tobacco smoke with vapour. ${ }^{13}$

\section{Smoke toxicants conversion}

Taking $\mathrm{N}$ to be the number of puffs in a smoking experiment, $\mathrm{S}$ the puff volume and D the filter ventilation (ie, fraction of air flow through filter vents), then

$$
V_{k, i}^{*}=N_{k, i} S_{k}\left(1-D_{k}\right)
$$

where $V_{k, i}^{*}$ represents the undiluted smoke volume and $k$ is the machine smoking protocol for the $i$ th sample (cigarette). For the ISO protocol $\mathrm{S}=35 \mathrm{~mL}$ and $\mathrm{D}$ ranges from 0 to 1 , for $\mathrm{MDPH}$ $\mathrm{S}=45 \mathrm{~mL}$ and $\mathrm{D}$ is half the filter ventilation, and for the HCI protocol $\mathrm{S}=55 \mathrm{~mL}$ and $\mathrm{D}=0$.

The usefulness of the $\mathrm{V}^{*}$ parameter is illustrated in online supplementary figure $\mathrm{S} 1$ in which nicotine concentrations from the various smoking machine experiments are plotted against $V_{k, i}^{*}{ }^{14}$ The graph shows strong correlation between undiluted smoke volume $\mathrm{V}^{*}$ and nicotine concentration per cigarette $\left(r^{2}=0.93, p<0.01\right)$ with a linear regression intercept close to the origin. Similar co-linearity of $\mathrm{V}^{*}$ over the major carcinogens under consideration provides a means of expressing smoke carcinogens as concentrations in a form independent of machine smoking protocol.

$$
E_{k, i, j}=C_{k, i, j}^{t o b} / V_{k, i}^{*}
$$

where $C_{k, i, j}^{t o b}$ ( $\mu \mathrm{g} /$ cigarette) is the mass per cigarette of the $j t h$ carcinogen in the ith product that has been machine-smoked using protocol $k . E_{k, i, j}$ is the carcinogen concentration in units of $\mu \mathrm{g} / \mathrm{mL}$ in undiluted smoke. This transformation expresses tobacco smoke toxicants as concentrations in the product, in common with ECs. Furthermore, the denominator $\mathrm{V}^{*}$ is a good proxy for nicotine (see online supplementary figure S1). HnB emissions are treated in the same way as tobacco smoke. Toxicants in EC vapours $\left(C_{k, i, j}^{v a p}\right)$, usually reported as mass/volume concentrations, only need conversion to $\mu \mathrm{g} / \mathrm{mL}$, as appropriate.

\section{Aerosol cancer potency}

Environmental protection agencies assess toxicological, epidemiological and other data relevant to the long-term risks of inhaling chemicals. ${ }^{15}$ Unit risk values for cancer have been published where data are deemed adequate. ${ }^{16}$ The inhalation unit risk ( $\left.U_{j}\right)$ for the $j t h$ carcinogen is defined as the excess lifetime cancer risk from continuous inhalation exposure to $1 \mu \mathrm{g}$ of the carcinogen per $\mathrm{m}^{3}$ of air and is expressed in $\left(\mu \mathrm{g} / \mathrm{m}^{3}\right)^{-1} \cdot 15$

The cancer potency from the mixture of carcinogens in tobacco smoke may be estimated using an aggregate model that weights each inhalation unit risk by its concentration in undiluted smoke

$$
P_{k, i}^{t o b}=\sum_{j=1}^{m} E_{k, i, j} U_{j}
$$

Similarly, the cancer potency of EC vapour can be formulated as

$$
P_{i}^{v a p}=\sum_{j=1}^{m} C_{i, j}^{v a p} U_{j}
$$

$P^{t o b}$ and $P^{v a p}$ are aggregates of individual compound potencies and are measures of the carcinogenic potential of a given aerosol. The normalised ratio

$$
P_{i}^{N}=P_{i} / \bar{P}^{t o b}
$$

is the cancer potency of smoke or vapour relative to average tobacco smoke and is the dimensionless parameter used to discuss relative potency below.

\section{Modelling exposure and risk}

Metrics of exposure that reflect human use patterns are needed to translate potencies into risk estimates for each nicotine product. ${ }^{17}$ Studies of smoking usually report the number of cigarettes per day. Analogous reports of EC vaping topographies include estimates of vapour volume inhaled per day. Vaping is associated with greater volumes than tobacco smoking, possibly resulting in greater relative risks than implicit in the potency spectrum. $^{17}$

The lifetime cancer risk $\left(L_{i}^{t o b}\right)$ for the ith cigarette based on daily smoke exposure (assuming HCI conditions) takes into account the mean daily cigarettes in stick units (d) and the daily breathing rate of air (b), typically $20 \mathrm{~m}^{3}$,

$$
L_{i}^{t o b}=\frac{\sum_{j=1}^{m} C_{H C I, i, j}^{t o b} U_{j}}{b} d
$$

The same procedure is applied to $\mathrm{HnB}$ tobacco sticks.

For EC, the lifetime cancer risk $\left(L_{i}^{v a p}\right)$ for the $i$ th product is the aerosol potency scaled by the average daily volume of vapour inhaled $\bar{V}_{\text {day }}^{\text {vap }}$,

$$
L_{i}^{v a p}=P_{i}^{v a p} \frac{\bar{V}_{d a y}^{v a p}}{b}
$$

The mean daily number of cigarettes smoked was 13 in 22 countries during 2008-2014. ${ }^{18}$ In 2016, the average daily consumption in the UK was 12 cigarettes, whereas in the USA in 2015 the comparable figure was $14 .{ }^{19}{ }^{20}$ For this study, cigarettes per day were rounded to 15 , reflecting the low daily smoking in these countries. The same number is applied to $\mathrm{HnB}$ modelling, consistent with exposure studies in Japan (average 10 sticks/day) and Poland (average 17). ${ }^{21} 22$

The average daily volume of EC vapour inhaled $\left(\bar{V}_{\text {day }}^{\text {vap }}\right)$ has been reported as $29.7 \mathrm{~L} /$ day based on measurements using 22 subjects over a 24-hour period, but this result is associated with considerable variability (SD 29.6). ${ }^{23}$ A daily volume of $30 \mathrm{~L}$ is used here for risk modelling while recognising the large uncertainty involved. It has also been estimated that about four times greater volume of vapour is inhaled than tobacco smoke and this is broadly consistent with the adopted values. ${ }^{24}$ This volume falls within the range of daily nicotine inhaler use. ${ }^{25}$

\section{Data sources}

Eleven compounds commonly reported in tobacco smoke are classified by the International Agency for Research on Cancer (IARC) as human carcinogens (type 1) and a further seven as possible human carcinogens (type $2 \mathrm{~B}$ ). The inhalation unit risks for 14 of these carcinogens were obtained from the Office of Environmental Health Hazard Assessment database (OEHHA, California, USA) supplemented with a recommended value for the carcinogenic nitrosamine 4-(methylnitrosamino)-1-(3-pyridyl)-1-butanone (NNK) (table 1). ${ }^{26}$

Concentrations of several major carcinogens in cigarette smoke and in VNP vapour were obtained from studies of cigarettes, ${ }^{142728}$ a prototype $\mathrm{HnB}$ device ${ }^{29}$ and EC products, ranging from early-generation disposables through second-generation clearomisers and cartomisers to third-generation mods and tanks. ${ }^{70-37}$ EC coil resistance and battery voltage were collated where reported. This compilation has many gaps where some carcinogens were not analysed. After removing duplicates across studies and omitting non-steady-state emissions (where stated), 
Table 1 Unit risks and average concentrations for IARC type 1 and 2 carcinogens measured in tobacco smoke and other forms of nicotine delivery

\begin{tabular}{|c|c|c|c|c|c|c|}
\hline \multicolumn{3}{|l|}{ Carcinogens } & \multirow{2}{*}{$\begin{array}{l}\begin{array}{l}\text { Tobacco smoke } \\
(\mathrm{n}=309)\end{array} \\
\\
\text { Mean concentration } \\
\mathrm{E}_{\mathrm{j}}(\mu \mathrm{g} / \mathrm{mL})\end{array}$} & \multirow{2}{*}{$\begin{array}{l}\begin{array}{l}\text { Heat-not-burn } \\
\text { emission }(n=44)\end{array} \\
\text { Mean concentration } \\
E_{j}(\mu \mathrm{g} / \mathrm{mL})\end{array}$} & \multirow{2}{*}{$\begin{array}{l}\text { E-cigarette vapour }(n=44) \\
\text { Mean concentration in } \\
\text { first-generation and second- } \\
\text { generation e-cigarettes } C_{j}(\mu g / \\
m L)\end{array}$} & \multirow{2}{*}{$\begin{array}{l}\text { Nicotine inhaler }(\mathrm{n}=1) \\
\text { Mean concentration } C_{j} \\
(\mu \mathrm{g} / \mathrm{mL})\end{array}$} \\
\hline Compound & IARC type & $\begin{array}{l}\text { OEHHA unit risk } U_{j} \\
\left(\mu \mathrm{g} / \mathrm{m}^{3}\right)^{-1}\end{array}$ & & & & \\
\hline Acetaldehyde & $2 B$ & $2.7 \times 10^{-6}$ & $2.55 \times 10^{-0}$ & $3.33 \times 10^{-1}$ & $4.41 \times 10^{-3}$ & $1.05 \times 10^{-4}$ \\
\hline Formaldehyde & 1 & $6.0 \times 10^{-6}$ & $1.54 \times 10^{-1}$ & $1.06 \times 10^{-2}$ & $8.07 \times 10^{-3}$ & $1.90 \times 10^{-4}$ \\
\hline Acrylonitrile & $2 B$ & $2.9 \times 10^{-4}$ & $4.59 \times 10^{-2}$ & $2.96 \times 10^{-4}$ & NR & NR \\
\hline Benzene & 1 & $2.9 \times 10^{-5}$ & $1.57 \times 10^{-1}$ & $9.32 \times 10^{-4}$ & NR & NR \\
\hline 1,3-Butadiene & 1 & $1.7 \times 10^{-4}$ & $1.83 \times 10^{-1}$ & $3.94 \times 10^{-4}$ & NR & NR \\
\hline 2-Amino-naphthalene & 1 & $5.14 \times 10^{-4}$ & $4.13 \times 10^{-5}$ & $4.82 \times 10^{-8}$ & NR & NR \\
\hline 4-Amino-biphenyl & 1 & $6.0 \times 10^{-3}$ & $8.68 \times 10^{-6}$ & $1.80 \times 10^{-8}$ & NR & NR \\
\hline Benzo[a]pyrene & 1 & $1.1 \times 10^{-3}$ & $3.67 \times 10^{-5}$ & $2.12 \times 10^{-6}$ & NR & NR \\
\hline NNN & 1 & $4.0 \times 10^{-4}$ & $4.63 \times 10^{-4}$ & $2.57 \times 10^{-5}$ & $1.94 \times 10^{-7}$ & BDL \\
\hline NNK & 1 & $4.0 \times 10^{-4}$ & $2.88 \times 10^{-4}$ & $1.64 \times 10^{-5}$ & $8.39 \times 10^{-7}$ & $\mathrm{BDL}$ \\
\hline Cadmium & 1 & $4.2 \times 10^{-3}$ & $1.99 \times 10^{-4}$ & $\mathrm{BDL}$ & $1.01 \times 10^{-5}$ & $9.52 \times 10^{-7}$ \\
\hline Lead & $2 B$ & $1.2 \times 10^{-5}$ & $7.52 \times 10^{-5}$ & $4.09 \times 10^{-6}$ & $7.06 \times 10^{-6}$ & $1.90 \times 10^{-6}$ \\
\hline Chromium & 1 & $1.5 \times 10^{-1}$ & $\mathrm{BDL}$ & $\mathrm{BDL}$ & NR & NR \\
\hline Nickel & $2 B$ & $2.6 \times 10^{-4}$ & $\mathrm{BDL}$ & $\mathrm{BDL}$ & $6.98 \times 10^{-6}$ & $1.90 \times 10^{-6}$ \\
\hline Arsenic & 1 & $3.3 \times 10^{-3}$ & $2.20 \times 10^{-5}$ & $2.14 \times 10^{-6}$ & NR & NR \\
\hline \multicolumn{3}{|c|}{ Mean cancer potency ratio (equation 5) } & 1.0 & $2.01 \times 10^{-2}$ & $1.81 \times 10^{-3}$ & $1.02 \times 10^{-4}$ \\
\hline \multirow{4}{*}{\multicolumn{2}{|c|}{$\begin{array}{l}\text { Mean lifetime cancer risk (equations } \\
6 \text { and 7) }\end{array}$}} & Consumption & 15 cigarettes/day & 15 sticks/day & 30 L vapour/day & 30 L vapour/day \\
\hline & & Risk & $2.4 \times 10^{-2}$ & $5.7 \times 10^{-4}$ & $9.5 \times 10^{-5}$ & $8.9 \times 10^{-6}$ \\
\hline & & $\begin{array}{l}\text { Ratio to tobacco } \\
\text { smoke }\end{array}$ & 1.0 & 0.024 & 0.004 & 0.0004 \\
\hline & & $\begin{array}{l}\text { Ratio to nicotine } \\
\text { inhaler }\end{array}$ & 2697 & 64 & 10.7 & 1.0 \\
\hline
\end{tabular}

Average concentrations for e-cigarettes for first-generation and second-generation devices only (where identifiable). See text for sources of data. Concentrations in bold type represent compounds that contribute $>5 \%$ of the modelled potency for a particular form of delivery.

BDL, below detection limit; IARC, International Agency for Research on Cancer; NR, not reported; NNN, N'-nitrosonornicotine; NNK, 4-(methylnitrosamino)-1-(3-pyridyl)-1butanone; OEHHA, Office of Environmental Health Hazard Assessment.

the resulting data set contained 93 analyses divided into three subsets. $^{34}$ The Goniewicz subset is the benchmark with 12 EC samples analysed for seven carcinogens including carbonyls, volatile organic compounds (VOCs), nitrosamines and metals. ${ }^{7}$ The remaining 81 analyses include concentrations for some organic compounds (formaldehyde, acetaldehyde and occasionally VOCs) and thus yield minimum estimates of potency, of which 32 involved coil heating experiments applying multiple powers to the same liquid in the same device (variable power subset). The remainder $(n=48)$ are termed the organics-only subset.

Carcinogen emissions from an unheated nicotine inhaler represent a device accepted for medical use. ${ }^{7}$ Ambient air is represented by a suite of analyses that establish a relatively uncontaminated reference baseline for studies of smoke and vapour exposure. ${ }^{38}$

\section{RESULTS}

\section{Distribution of cancer potencies}

Cancer potencies were computed for the data sets using equations 3 and 4 following conversion into compatible units of concentrations (equations 1 and 2) and matched with unit risks for cancer. The calculation sequence is summarised in online supplementary table $\mathrm{S} 1$. Average concentrations, unit risks and cancer potencies for various nicotine products are given in table 1 , and the potencies of individual samples are graphically presented in figure 1. All results are normalised to the average value for tobacco smoke and plotting the results on a logarithmic axis creates a relative cancer potency spectrum for the various nicotine-bearing aerosols.

Smoke collected under the ISO protocol and converted to undiluted smoke volume $V^{*}$ has essentially the same potency as that collected under HCI (figure 1). Without factoring for filter ventilation, the ISO samples would be widely dispersed ranging down to much lower apparent cancer potencies for the most ventilated brands. HCI and ISO tobacco smoke have the same distributions after applying the $V^{*}$ conversion (figure 1 ) which indicates that raw undiluted tobacco smoke occupies a narrow band on the potency spectrum regardless of protocol and defines the upper boundary of cancer potencies among common nicotine products in high-income countries.

Ambient air, essentially a non-hazardous aerosol, defines the lower end of the cancer potency spectrum, clustering around a carcinogenic potency of slightly less than $10^{-5}$ that of tobacco smoke. The potency of the nicotine inhaler is about $10^{-4}$ that of tobacco smoke (figure 1), with metals contributing $>70 \%$ of its carcinogenic potency.

The cancer potencies of EC are more difficult to define and are highly dispersed compared with cigarettes. The Goniewicz subset, which analyses the majority of anticipated carcinogens in EC vapour, clusters around $\sim 10^{-3}$ of the potency of tobacco smoke (figure 1). The preponderance of potencies in the organics-only and variable power subsets also falls in this range, although higher levels of carbonyls found in these two subsets extend the range as far as the potency of tobacco smoke. 


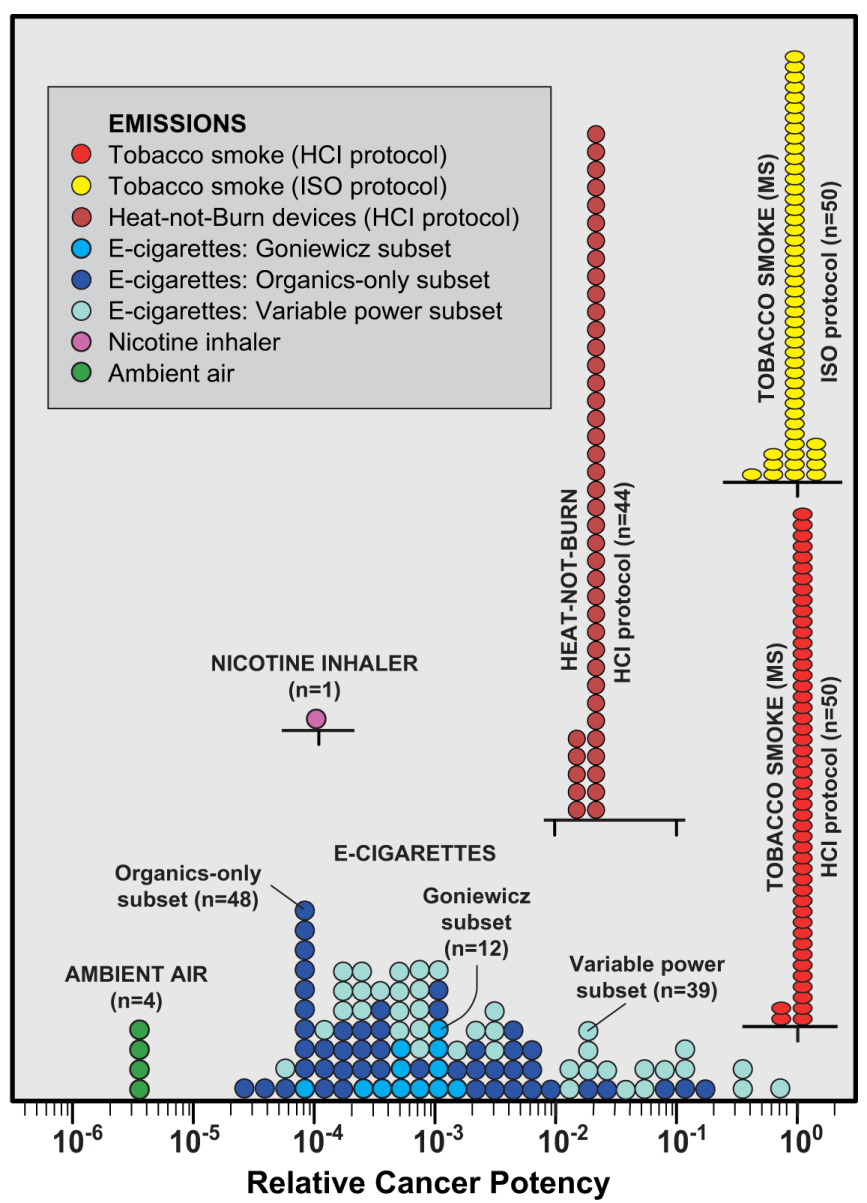

Figure 1 Relative cancer potency modelled for common forms of nicotine delivery. Potency is the aggregate estimated using equations 3 and 4 and normalised to average tobacco smoke with cancer risk set to 1.0 (equation 5). Each circle or ellipse on the graph represents the emission from a single sample or experiment in the peer reviewed literature. In all, emissions from 14 peer-reviewed studies are summarised in the diagram (see text for data sources). MS, mainstream smoke.

The cancer potencies of the $\mathrm{HnB}$ prototype device that heated various tobacco blends lie between one and two orders of magnitude less than tobacco smoke but higher than the preponderance of EC emissions (figure 1).

\section{Lifetime cancer risk}

Equations 6 and 7 estimate the lifetime cancer risks from daily exposure (table 1). The values for ECs in this table are based on a compilation of first-generation and second-generation devices because most third-generation tanks and mods were used for variable voltage experiments. The average for EC includes the Goniewicz subset supplemented by carbonyl analyses from those members of the organic-only subset for which the device generation could be identified. The potency ratio relative to tobacco smoke for the average of these ECs is $1.8 \times 10^{-3}$ while $\mathrm{HnB}$ had an order of magnitude higher relative potency at $2.1 \times 10^{-2}$. The excess cancer risk for a lifetime of smoking 15 cigarettes a day is $2.4 \times 10^{-2}, 5.7 \times 10^{-4}$ for inhaling $15 \mathrm{HnB}$ sticks, $9.5 \times 10^{-5}$ for vaping $30 \mathrm{~L}$ from ECs at normal power and $8.9 \times 10^{-6}$ for inhaling $30 \mathrm{~L}$ from the nicotine inhaler (table 1). Compared with a nicotine inhaler, the relative risks are 11 for EC, 64 for $\mathrm{HnB}$ and $\sim 2700$ for cigarettes, whereas compared with cigarettes the relative risks are 0.024 for $\mathrm{HnB}, 0.004$ for $\mathrm{EC}$ and 0.0004 for the nicotine inhaler (table 1 ).

\section{Ranking carcinogens}

The highest-ranking carcinogens in table 1 for cigarettes are 1,3-butadiene and acrylonitrile, accounting for more than three-quarters of the cancer potency, whereas for HnB, acetaldehyde is the dominant carcinogen, which, along with 1,3-butadiene, accounts for almost three-quarters of the aerosol's potency. For ECs, only the Goniewicz subset includes nitrosamines and metals as well as carbonyls and VOCs. The highest ranked carcinogens in this subset are cadmium and formaldehyde. Even very small levels of cadmium can have a major effect given its extremely high unit risk (table 1) but cadmium is not detected in all samples and in others it is present in concentrations very close to blank level. ${ }^{7}$ Formaldehyde and acetaldehyde account for $>95 \%$ of the contributions of organic compounds to cancer potencies in this EC subset.

\section{Role of carbonyls in the cancer potency of EC vapour}

Given the importance of the carbonyls as carcinogens in EC vapour, the relationship between battery voltage and carbonyl generation is explored further in figure 2. Several studies have addressed this issue by measuring carbonyl concentrations under various experimental conditions. ${ }^{3132343739}$ These were omitted from the modelling of potency and risk of table 1 because they often involve applying voltages beyond the normal range of use. This figure shows that the partial cancer potencies attributable to both formaldehyde and acetaldehyde are highly variable. The Goniewicz subset and some other samples from both of the other subsets represent the lower range of potencies attributable to carbonyls. Other data indicate considerably higher potencies due to both carbonyls, sometimes approaching and, for formaldehyde, even exceeding the partial potencies of carbonyls in tobacco smoke (figure 2). Most of the high-potency data points in figure 2 were from studies investigating carbonyl formation by varying the atomiser coil heat within the same device while varying the power (watts) at the fluid-coil interface. Solid lines in figure 2 connect the various powers achieved in the same experiment with the direction of increasing power indicated by an arrow. The highest cancer potencies are commonly associated with coils subjected to the highest applied voltages in any particular experiment; however, there is no consistent relationship between devices for carbonyl potency and the rate of heat energy transfer (watts) at the coil. It has been suggested that such high-power operating conditions produce 'dry puffs', that is, when the supply of e-liquid to the heating coil is inadequate producing an aerosol which is unpleasant to inhale although an exclusive causal relationship between dry puffs and high aldehyde concentrations has been questioned. ${ }^{40} 41$

\section{DISCUSSION}

\section{Strengths and limitations of emissions modelling}

The accuracy of emissions modelling can be evaluated by comparing the lifetime risk model for tobacco smoking with epidemiological data for smoking-related mortality. An early application of this model to tobacco smoke predicted an excess risk of $1.6 \times 10^{-2}$ for a daily consumption of 20 cigarettes measured with the ISO protocol. ${ }^{26}$ This differed from the estimated excess cancer risk of $7.9 \times 10^{-2}$ derived from mortality data for smokers in the USA in $1995 .{ }^{14}$ Applying the same model to the Centers for Disease Control tobacco smoke data set 


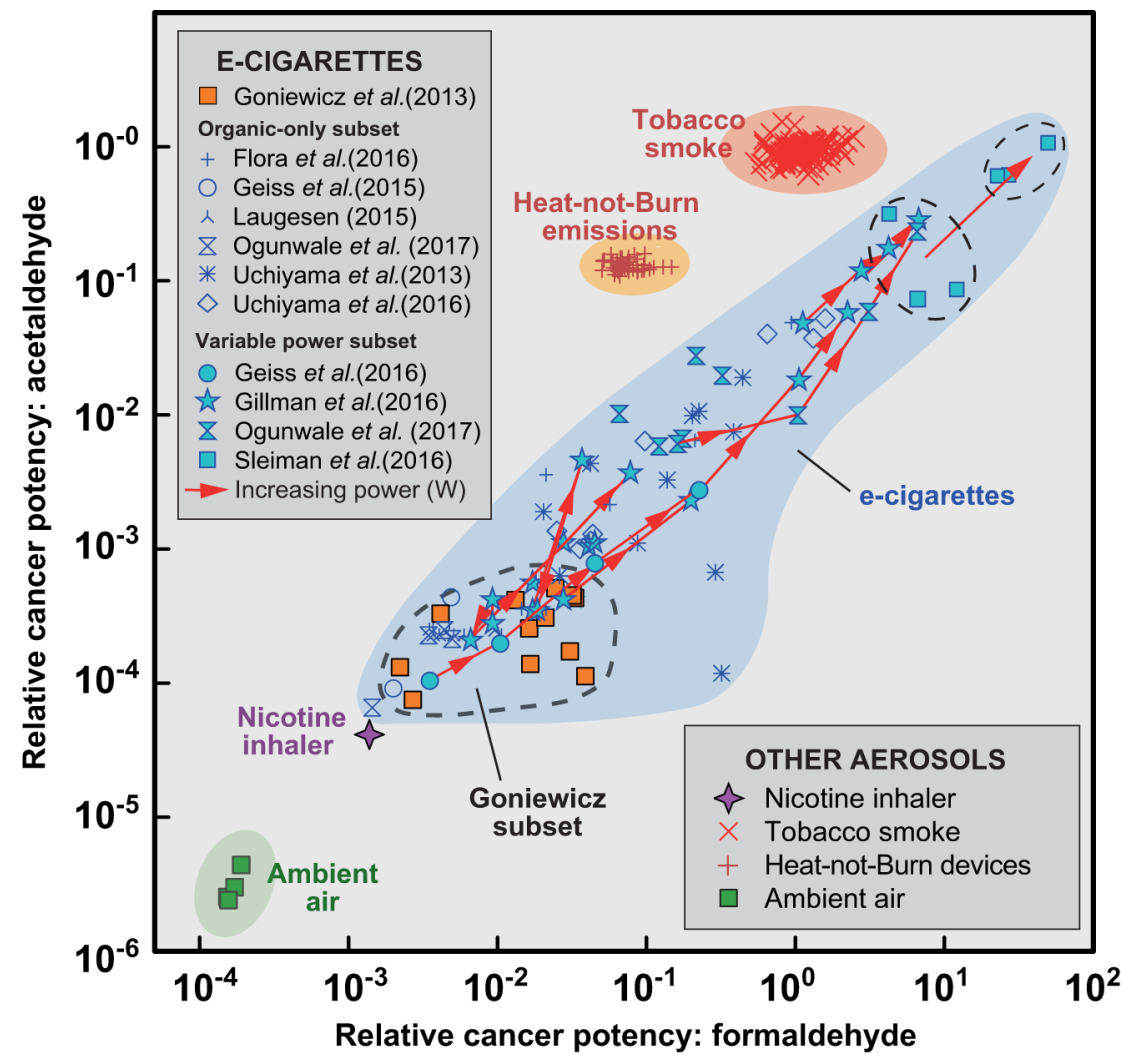

Figure 2 Relative cancer potencies of formaldehyde and acetaldehyde in vapour from e-cigarettes compared with tobacco smoke, heat-not-burn devices, a nicotine inhaler and ambient air. Values are normalised to the mean potency of the same carcinogens in tobacco smoke. Data sources for e-cigarettes are identified with different symbols (see key for sources). The variable power subset includes all devices used in experiments to create vapour at more than one atomiser power (watts). Solid red lines connect the potencies of emissions for the same device run at different powers with arrows indicating the direction of increasing power. The lines show that the cancer potencies from the same device can vary by more than two orders of magnitude for both formaldehyde and acetaldehyde.

measured with the HCI protocol weighted by current OEHHA unit risk values doubles the estimated risk to $3.3 \times 10^{-2}$ averaged over 48 cigarette products (SD $3.5 \times 10^{-3}$ ) for the same daily consumption rate. This closer agreement is largely due to the use of the HCI machine smoking protocol for emissions, suggesting that these independent estimates reflect the same fundamental causes of mortality.

Using emissions can underestimate and overestimate risk. Aggregation of risks for individual carcinogens assumes that each inhaled carcinogen is wholly absorbed and no account is taken of differential mechanisms and rates of clearance. Unit risks are upper bound toxicological estimates that may overestimate true potency. ${ }^{42}$ If large volumes of carbonyls are generated by EC use under circumstances not normally tolerated by humans (eg, 'dry puffs'), then the apparent high-risk levels may not be reached. ${ }^{40}$

A simple aggregation does not account for enhancement by synergystic interactions between components (but there may also be antagonistic interactions). A few synergystic effects are well known, often between tobacco smoke and particles such as asbestos and silica, but these are largely restricted to industrial exposure (although silica particles have been recognised in EC vapour). ${ }^{43}$ Unit risk values are not available for all known carcinogens and are thus not involved in the aggregation leading to underestimation. Furthermore, only carcinogens formally classified by the IARC are included. Although cigarette smoke has been thoroughly investigated for toxicants over several decades, EC have not received the same level of scrutiny and some carcinogens are possibly yet to be identified, although these 'missing carcinogens' would need to be present in high concentrations and/or have high unit risks to make a noticeable difference to EC potency and risk. Other sources of uncertainties are differences in unit risks between environmental protection authorities, and the assumption that risk is linearly proportional to dose with no threshold.

Another limitation is the absence of carcinogen analyses in most published studies of EC vapours. Only the Goniewicz subset presents data for most of the relevant carcinogens, and these indicate very important roles for metals (especially cadmium) and formaldehyde. The importance of cadmium as a carcinogen in vapour needs further investigation as it has not been detected at hazardous levels in other studies of vapour. ${ }^{44-46}$ Also missing from most studies are analyses of the nitrosamine carcinogens, notably $\mathrm{NNK}$ and $\mathrm{N}^{\prime}$-nitrosonornicotine (NNN), although potencies calculated from the Goniewicz subset suggest these are minor contributors to the risk (table 1). Indeed missing data for carcinogens at low concentrations or with low unit risks may have little or no significant effect on modelled potency and risk. Note that particle size effects have not been taken into account in this or previous chemical studies of this type but are potentially significant. ${ }^{47} 48$ 
VNP and cigarette emissions have been compared in this study using carcinogen concentrations, whereas normalisation to unit nicotine levels is sometimes preferred as an estimate of human exposure. ${ }^{49}$ Nicotine has an unprotonated (free-base) and two protonated forms, unprotonated nicotine being of greatest concern in terms of blood transfer. The fraction of total nicotine in free-base form varies widely in both tobacco smoke and EC vapour largely as a function of $\mathrm{pH} .{ }^{5051}$ Whether free-base nicotine could be a useful proxy for exposure in tobacco-based aerosols requires further research as does the relationship between the concentrations of the various nicotine forms and exposure to toxicants in EC emissions.

Biomarkers for 1,3-butadiene and acrylonitrile in urine samples from EC-only users indicate levels similar to nicotine replacement therapy users, in contrast to the high levels found in smokers. ${ }^{10}$ Biomarkers for the same compounds declined rapidly in smokers switching to ECs at rates similar to abstinence from smoking over 5 days. ${ }^{52}$ Neither compound is normally detectable in EC vapour yet both have been shown above to account collectively for more than three-quarters of the cancer potency of tobacco smoke. ${ }^{53}$ This close correlation between reduced emissions in EC vapours and their associated biomarkers for two of the most important carcinogens in tobacco smoke supports the use of emissions in estimating potency and risk.

\section{Carbonyl generation}

The preponderance of EC analyses conducted at fixed or recommended voltages indicates relatively low risks of exposure related to carbonyls (figure 2 and table 1), notwithstanding, additional carbonyl generation at higher powers has a major influence on cancer potency (figure 2). While factors such as the propylene glycol/glycerol balance and other e-liquid ingredients as well as puff number may influence aldehyde formation, overheating appears to dominate. ${ }^{34} 54-56$ Indeed, the connected arrows on figure 2 indicate that up to half the cancer potency range for ECs can be generated from the same liquid in a single device by varying only the power. This implies that the way a device is used might be more important than the device itself. The unpleasant sensory experience of a 'dry puff' may mean that these laboratory-derived data points are replicated less frequently in normal human exposure than implied by the machine smoking data presented in figure $2 .^{40}$

\section{Heat-not-burn devices}

Data from experiments on one $\mathrm{HnB}$ device indicate cancer potencies and risks lower than tobacco smoke by more than one order of magnitude greater than the preponderance of ECs operating under normal conditions (table 1). This finding is provisional as it is based on a single prototype design from a single manufacturer.

\section{Caveats}

Unit risk values (table 1 ) indicate a potentially major role for metals even at low concentrations. ECs are typically metallic devices, not least the atomiser which is normally a metallic coil usually made of nichrome ( $\mathrm{NiCr}$ alloy) or kanthal (FeCrAl alloy). Other metallic components can include electrical conductors, crimps, connectors, solder, cartridges and structural features of the device, many of which show some evidence of corrosion over time in prefilled devices. Several papers document elevated levels of metals in liquids and occasionally the aerosol but no consistency is yet evident in emissions. ${ }^{7445} 57$ The lack of metals data for samples other than the Goniewicz subset means that some cancer potency values for ECs in this study may be underestimated. Even less is known about the speciation of metals in EC vapour despite the importance of different valence states and molecular speciation in tobacco smoke toxicity. ${ }^{58} 59$ More detailed study of metals, especially $\mathrm{Ni}, \mathrm{Cr}$ and $\mathrm{Pb}$ and their speciation in a wider of range of products, may lead to new perspectives on the cancer potential of metals in EC vapours.

The presence of fine and nanosize particulates in EC vapour was recently demonstrated. ${ }^{48}$ Given the greater surface area and potential reactivity of nanoparticles, if their presence in VNP vapours is confirmed as substantial then safety estimates for these products may require re-evaluation, especially in relation to cancer, respiratory and cardiovascular diseases including stroke. ${ }^{60}$

The cancer potency of secondhand vapour was not specifically addressed in this study. The few published experiments of secondhand vaping suggest low cancer potencies, although none appeared to involve products that emitted relatively high levels of carbonyls in the mainstream vapour. ${ }^{61} 62$

\section{Implications for policy and practice}

The data imply a considerable range of cancer risks. Many EC emissions have cancer potencies within an order of magnitude of a nicotine inhaler, a product generally regarded as safe. Notwithstanding, some EC emissions tended towards much higher cancer potencies and risks, a few possibly approaching those of tobacco smoke. Indeed, the cancer potency of formaldehyde, the most important EC carcinogen, can exceed that of tobacco smoke especially in the highest power settings of some multipower experiments but the full range of relative potency spans nearly four orders of magnitude with the vast majority of potencies being much lower than combustible cigarettes. It is likely that third-fourth-generation EC devices with adjustable coil power are implicated in these higher risks. With regard to involuntary exposure, better understanding of potential effects of secondhand EC exposure is needed to determine if their use in indoor public spaces should be banned, as is currently the case in 25 countries. $^{63}$

The conclusions of this study refer only to the chemical risks of cancer and do not account for any other carcinogenic effects such as those attributable to small particle sizes. There is some evidence that the large differentials found here between the cancer potencies of most ECs and those of tobacco smoke may be less for other medical conditions involving the cardiovascular and respiratory systems. ${ }^{37}$ Future research should address these gaps.

The cancer potency calculation for VNPs was applied to a range of devices covering different generations purchased in different countries but no claim is made that these samples are representative of all products in the current marketplace. As the VNP market continues to expand and diversify, a better understanding of the origins and aerosol transfer of carbonyls and metals is required to achieve EC emissions with consistently low carcinogenic potency. Such understanding may prompt calls for constraints on device design and e-liquid formulations but it is likely that significant reduction in cancer risk could also be achieved by effective advice to EC users on generic aspects of devices, coil selection and refill liquids as well as vaping behaviour and product manipulation.

Competing interests None declared 


\section{What this paper adds}

- Emissions from vapourised nicotine products (VNP) including e-cigarettes contain carcinogens but generally in lower concentrations than tobacco smoke.

- Each carcinogen contributes quantifiably to the overall cancer potency and risk.

- Users and policymakers need quantitative evidence on the relative risks of cancer from the use of VNPs compared with smoking tobacco.

- Previous studies considered the individual carcinogens in an emission; here a method is developed that models the aggregate cancer potencies of all measured carcinogens and overcomes incompatibilities in data reporting conventions enabling direct comparison of the potencies and risks of tobacco smoke with VNP emissions.

- Cancer potencies span five orders of magnitude creating a spectrum ranging from uncontaminated air through VNPs to tobacco smoke.

- Most e-cigarette analyses indicate cancer potencies $<1 \%$ that of tobacco smoke and $<10 \%$ that of a heat-not-burn prototype, although a minority of analyses indicate higher potencies.

- Highly carcinogenic emissions from e-cigarettes are avoidable, being due largely to user choice of device setting, liquid formulation and vaping behaviour, highlighting a need for increased user awareness and personal involvement in reducing risk.

Provenance and peer review Not commissioned; externally peer reviewed. (c) Article author(s) (or their employer(s) unless otherwise stated in the text of the article) 2018. All rights reserved. No commercial use is permitted unless otherwise expressly granted.

\section{REFERENCES}

1 Green SH, Bayer R, Fairchild AL. Evidence, Policy, and E-Cigarettes--Will England Reframe the Debate? N Engl J Med 2016;374:1301-3.

2 Smith MR, Clark B, Lüdicke F, et al. Evaluation of the tobacco heating system 2.2. Part 1: Description of the system and the scientific assessment program. Regul Toxicol Pharmacol 2016;81(Suppl 2):S17-26.

3 ASH. Use of electronic cigarettes (vapourisers) among adults in great Britain. Fact Sheet: Action on Smoking and Health (ASH), 2016:1-13.

4 Huerta TR, Walker DM, Mullen D, et al. Trends in E-cigarette awareness and perceived harmfulness in the U.S. Am J Prev Med 2017;52:339-46.

5 Czoli CD, Fong GT, Mays D, et al. How do consumers perceive differences in risk across nicotine products? A review of relative risk perceptions across smokeless tobacco, e-cigarettes, nicotine replacement therapy and combustible cigarettes. Tob Control 2017;26(e1):e49-58.

6 Chen J, Bullen C, Dirks K. A comparative health risk assessment of electronic cigarettes and conventional cigarettes. Int I Environ Res Public Health 2017;14:382.

7 Goniewicz ML, Knysak J, Gawron M, et al. Levels of selected carcinogens and toxicants in vapour from electronic cigarettes. Tob Control 2014;23:133-9.

8 Farsalinos KE, Voudris V, Poulas K. Are metals emitted from electronic cigarettes a reason for health concern? A risk-assessment analysis of currently available literature. Int J Environ Res Public Health 2015;12:5215-32.

9 Hiemstra PS, Bals R. Basic science of electronic cigarettes: assessment in cell culture and in vivo models. Respir Res 2016;17:5.

10 Shahab L, Goniewicz ML, Blount BC, et al. Nicotine, carcinogen, and Toxin Exposure in Long-Term E-Cigarette and Nicotine Replacement therapy users: a Cross-sectional study. Ann Intern Med 2017;166:390-400.

11 Anon. The health consequences of smoking - 50 years of progress: a report of the Surgeon General. Atlanta, USA: U.S. Department of Health and Human Services, Centers for Disease Control and Prevention, National Center for Chronic Disease Prevention and Health Promotion, Office on Smoking and Health, 2014:944.

12 ISO. Routine analytical cigarette-smoking machine: definitions and standard conditions, 2012:25.
13 Stephens WE. Dependence of tar, nicotine and carbon monoxide yields on physical parameters: implications for exposure, emissions control and monitoring. Tob Control 2007;16:170-6.

14 Counts ME, Morton MJ, Laffoon SW, et al. Smoke composition and predicting relationships for international commercial cigarettes smoked with three machinesmoking conditions. Regul Toxicol Pharmacol 2005;41:185-227.

15 USEPA. Basic information about the Integrated Risk Information System, 2017.

16 USEPA. An Overview of Methods for EPA's National-Scale Air Toxics Assessment: US Environmental Protection Agency. Office of Air Quality, Planning, and Standards 2011.

17 Farsalinos KE, Romagna G, Tsiapras D, et al. Evaluation of electronic cigarette use (vaping) topography and estimation of liquid consumption: implications for research protocol standards definition and for public health authorities' regulation. Int J Environ Res Public Health 2013;10:2500-14

$18 \mathrm{NIH}$. The Economics of tobacco and tobacco control. Bethesda, MD: U.S and Geneva, $\mathrm{CH}$ : National Cancer Institute Tobacco Control Monograph and Department of Health and Human Services, National Institutes of Health, National Cancer InstituteWorld Health Organization, 2016.

19 Jamal A, King BA, Neff LJ, et al. Current cigarette smoking among adults - United States, 2005-2015. MMWR Morb Mortal Wkly Rep 2016;65:1205-11.

20 ONS. Adult smoking habits in the UK: 2016. Office for National Statistics, 2017:18.

21 Haziza C, de La Bourdonnaye G, Merlet S, et al. Assessment of the reduction in levels of exposure to harmful and potentially harmful constituents in Japanese subjects using a novel tobacco heating system compared with conventional cigarettes and smoking abstinence: A randomized controlled study in confinement. Regul Toxicol Pharmacol 2016;81:489-99.

22 Haziza C, de La Bourdonnaye G, Skiada D, et al. Evaluation of the tobacco heating system 2.2. Part 8: 5-day randomized reduced exposure clinical study in Poland. Regul Toxicol Pharmacol 2016:81(Suppl 2):S139-50.

23 Robinson RJ, Hensel EC, Morabito PN, et al. Electronic cigarette topography in the Natural Environment. PLoS One 2015;10:14.

24 Behar RZ, Hua M, Talbot P. Puffing topography and nicotine intake of electronic cigarette users. PLoS One 2015;10:18

25 Bolliger CT, Zellweger JP, Danielsson T, et al. Smoking reduction with oral nicotine inhalers: double blind, randomised clinical trial of efficacy and safety. BMJ 2000:321:329-33.

26 Fowles J, Dybing E. Application of toxicological risk assessment principles to the chemical constituents of cigarette smoke. Tob Control 2003;12:424-30.

27 Bodnar JA, Morgan WT, Murphy PA, et al. Mainstream smoke chemistry analysis of samples from the 2009 US cigarette market. Regul Toxicol Pharmacol 2012;64:35-42.

28 Pazo DY, Moliere F, Sampson MM, et al. Mainstream smoke levels of volatile organic compounds in 50 U.S. Domestic cigarette brands smoked with the ISO and Canadian intense protocols. Nicotine Tob Res 2016:18:1886-94.

29 Schaller JP, Pijnenburg JP, Ajithkumar A, et al. Evaluation of the tobacco heating system 2.2. Part 3: Influence of the tobacco blend on the formation of harmful and potentially harmful constituents of the tobacco heating system 2.2 aerosol. Regul Toxicol Pharmacol 2016;81(Suppl 2):S48-58

30 Flora JW, Wilkinson CT, Wilkinson JW, et al. Method for the determination of carbonyl compounds in e-cigarette aerosols. J Chromatogr Sci 2017;55:142-8.

31 Geiss O, Bianchi I, Barrero-Moreno J. Correlation of volatile carbonyl yields emitted by e-cigarettes with the temperature of the heating coil and the perceived sensorial quality of the generated vapours. Int J Hyg Environ Health 2016;219:268-77

32 Gillman IG, Kistler KA, Stewart EW, et al. Effect of variable power levels on the yield of total aerosol mass and formation of aldehydes in e-cigarette aerosols. Regul Toxicol Pharmacol 2016;75:58-65.

33 Laugesen M. Nicotine and toxicant yield ratings of electronic cigarette brands in New Zealand. N Z Med J 2015;128:77-82.

34 Sleiman M, Logue JM, Montesinos VN, et al. Emissions from electronic cigarettes: key parameters affecting the release of harmful chemicals. Environ Sci Technol 2016:50:9644-51.

35 Uchiyama S, Ohta K, Inaba Y, et al. Determination of carbonyl compounds generated from the E-cigarette using coupled silica cartridges impregnated with hydroquinone and 2,4-dinitrophenylhydrazine, followed by high-performance liquid chromatography. Anal Sci 2013;29:1219-22.

36 Uchiyama S, Senoo Y, Hayashida H, et al. Determination of Chemical compounds generated from Second-generation E-cigarettes using a Sorbent Cartridge followed by a Two-step Elution Method. Anal Sci 2016;32:549-55.

37 Ogunwale MA, Li M, Ramakrishnam Raju MV, et al. Aldehyde detection in electronic cigarette aerosols. ACS Omega 2017;2:1207-14

38 Oldham MJ, Wagner KA, Gene Gilman I, et al. Development/verification of methods for measurement of exhaled breath and environmental e-vapor product aerosol. Regul Toxicol Pharmacol 2017;85:55-63.

39 Kosmider L, Sobczak A, Fik M, et al. Carbonyl compounds in electronic cigarette vapors: effects of nicotine solvent and battery output voltage. Nicotine Tob Res 2014;16:1319-26.

40 Farsalinos KE, Voudris V, Poulas K. E-cigarettes generate high levels of aldehydes only in 'dry puff' conditions. Addiction 2015;110:1352-6. 
41 Khlystov A, Samburova V. Response to comment on "flavoring compounds dominate toxic aldehyde production during e-cigarette vaping". Environ Sci Technol 2017;51:2493-4.

42 Asante-Duah K. Public Health Risk Assessment for Human Exposure to Chemicals. Environmental Pollution: Springer, 2002.

43 De Matteis S, Consonni D, Lubin JH, et al. Impact of occupational carcinogens on lung cancer risk in a general population. Int J Epidemiol 2012;41:711-21.

44 Williams M, Villarreal A, Bozhilov K, et al. Metal and silicate particles including nanoparticles are present in electronic cigarette cartomizer fluid and aerosol. PLOS One 2013;8:e57987-11.

45 Palazzolo DL, Crow AP, Nelson JM, et al. Trace metals derived from Electronic Cigarette (ECIG) generated Aerosol: Potential problem of ECIG devices that contain nickel. Front Physiol 2016;7:17.

46 Visser W, Geraets L, Klerx W, et al. The health risks of using e-cigarettes. RIVM Letter report, 2015:47.

47 Hamra GB, Guha N, Cohen A, et al. Outdoor particulate matter exposure and lung cancer: a systematic review and meta-analysis. Environ Health Perspect 2014;122:906-11.

48 Mikheev VB, Brinkman MC, Granville CA, et al. Real-Time measurement of Electronic Cigarette Aerosol size distribution and Metals Content analysis. Nicotine Tob Res 2016;18:1895-902.

49 Benowitz NL, Gan Q, Goniewicz ML, et al. Different profiles of carcinogen exposure in Chinese compared with US cigarette smokers. Tob Control 2015;24:e258-63.

50 El-Hellani A, El-Hage R, Baalbaki R, et al. Free-Base and protonated nicotine in electronic cigarette liquids and aerosols. Chem Res Toxicol 2015;28:1532-7.

51 Pankow JF, Tavakoli AD, Luo W, et al. Percent free base nicotine in the tobacco smoke particulate matter of selected commercial and reference cigarettes. Chem Res Toxicol 2003;16:1014-8.

52 O'Connell G, Graff DW, D'Ruiz CD. Reductions in biomarkers of exposure (BoE) to harmful or potentially harmful constituents (HPHCs) following partial or complete substitution of cigarettes with electronic cigarettes in adult smokers. Toxicol Mech Methods 2016;26:453-64.

53 Tayyarah R, Long GA. Comparison of select analytes in aerosol from e-cigarettes with smoke from conventional cigarettes and with ambient air. Regul Toxicol Pharmacol 2014;70:704-10.

54 Farsalinos K, Gillman G, Kistler K, et al. Comment on "Flavoring Compounds Dominate Toxic Aldehyde Production during E Cigarette Vaping". Environ Sci Technol 2017;51:2491-2.

55 Wang P, Chen W, Liao J, et al. A Device-Independent evaluation of carbonyl emissions from heated electronic cigarette solvents. PLoS One 2017;12:e0169811.

56 Jensen RP, Strongin RM, Peyton DH. Solvent chemistry in the electronic cigarette reaction vessel. Sci Rep 2017;7:42549.

57 Hess CA, Olmedo P, Navas-Acien A, et al. E-cigarettes as a source of toxic and potentially carcinogenic metals. Environ Res 2017;152:221-5.

58 Campbell RC, Stephens WE, Meharg AA. Consistency of arsenic speciation in global tobacco products with implications for health and regulation. Tob Induc Dis 2014;12:8.

59 Campbell RC, Stephens WE, Finch AA, et al. Controls on the valence species of arsenic in tobacco smoke: XANES investigation with implications for health and regulation. Environ Sci Technol 2014;48:3449-56.

60 Shah AS, Lee KK, McAllister DA, et al. Short term exposure to air pollution and stroke: systematic review and meta-analysis. BMJ 2015;350:h1295.

61 McAuley TR, Hopke PK, Zhao J, et al. Comparison of the effects of e-cigarette vapor and cigarette smoke on indoor air quality. Inhal Toxicol 2012;24:850-7.

62 Czogala J, Goniewicz ML, Fidelus B, et al. Secondhand exposure to vapors from electronic cigarettes. Nicotine Tob Res 2014;16:655-62.

63 Kennedy RD, Awopegba A, De León E, et al. Global approaches to regulating electronic cigarettes. Tob Control 2017;26:440-5. 